\title{
ON SOME TOPOLOGICAL PROBLEMS ARISING FROM MAPS OF CONTRACTIVE TYPE
}

\author{
KOK-KEONG TAN ${ }^{1}$ AND CHI-SONG WONG ${ }^{2}$

\begin{abstract}
Characterizations of sequentially complete and of countably compact subsets of a uniform space are obtained. Applications to fixed point theory and coincidence theory are given.
\end{abstract}

0. Introduction. In 1969, the second author proved in [6] a fixed point theorem for a class $\mathcal{U}(X)$ of self-maps on a complete metric space $(X, d)$. This result generalizes the Banach contraction mapping theorem [9], a result of $F$. E. Browder [3], [8], and a result of M. Edelstein [5], [9]; by using a theorem in [7], [9], one can prove that it also generalizes a result of D. W. Boyd and J. S. W. Wong [2], [9]. Later in [9], [10] the above result was further generalized to self-maps on a complete Hausdorff uniform space.

In 1973, Dugundji introduced in [4] a notion of positive definiteness. It turns out that for a self-map $f$ on a metric space $(X, d), f \in \mathcal{U}(X)$ if and only if the map $P$ on $X \times X$ defined by $P(x, y)=d(x, T(x))+d(y, T(y)), x, y \in X$, is positive definite $\bmod \Delta(X)$, where $\Delta(X)$ is the diagonal of $X \times X$ [4]. Dugundji's notion of positive definiteness is useful since it can be used to characterize completeness and compactness of subsets of a metric space [4]. The notion of positive definiteness was soon generalized to uniform spaces in [10]. Since there are various equivalent notions of completeness and of compactness in metric spaces, it is interesting to determine if any of these equivalent notions can be characterized by the notion of positive definiteness in uniform spaces. The main purpose of this paper is to continue the effort made earlier in [10].

1. Main result. Let $(X, \mathcal{Q})$ be a uniform space, $A$ a subset of $X$ and $P$ a nonnegative real-valued function on $X$. For any $U$ in $\mathscr{Q}$, let

Received by the editors July 4, 1975 and, in revised form, July 11, 1976.

AMS (MOS) subject classifications (1970). Primary 54C30; Secondary 54H25.

Key words and phrases. Coincidence, compactness, completeness, countable compactness, fixed point, Hausdorff pseudo-metric, lower semicontinuity, positive definiteness, sequential completeness, uniformity.

${ }^{1}$ The author is partially supported by National Research Council of Canada under Grant A-8096.

2 The author is partially supported by National Research Council of Canada under Grant A-8518. 


$$
\delta(U)=\inf P(X-U[A]) \quad(\inf \varnothing=\infty) .
$$

$\delta$ is called the modulus function induced by $P$ and $A . P$ is positive definite $\bmod A$ if $\delta(U)>0$ for all $U$ in $थ$.

TheOrem 1. Let $(X, \mathcal{Q})$ be a Hausdorff uniform space. Then $(X, \mathcal{Q})$ is sequentially complete if and only if for any lower semicontinuous function $V$ of $X$ into $[0, \infty), V(x)=0$ for some $x$ in $X$ whenever inf $V(X)=0$ and the function $P_{V}$ on $X \times X$ defined by $P_{V}(x, y)=V(x)+V(y), x, y \in X, \cdot$ is positive definite $\bmod \Delta(X)$

PROOF. The "only if" part follows from the proof of Theorem 3 in [10], with "complete" being replaced by "sequentially complete". For the "if" part, let $\left\{F_{n}\right\}$ be a decreasing sequence of nonempty closed sets in $X$ which contains small sets. It suffices to show that $\cap_{n=1}^{\infty} F_{n} \neq \varnothing$. We may assume without loss of generality that all $F_{n}$ 's are distinct. Note that there exists a saturated family D of pseudometrics on $X$ such that $\mathscr{D}$ induces $\mathcal{U}$ and $d \leqslant 1$ for all $d \in \mathscr{D}$. Let $V$ be the function on $X$ defined by

$$
V(x)=\sup \left\{d\left(x, F_{n}\right) / n: d \in \mathscr{D}, n \geqslant 1\right\},
$$

for all $x$ in $X$. Then $V$ is a lower semicontinuous function of $X$ into $[0,1]$. We claim that inf $V(X)=0$. Indeed, for $\varepsilon>0$, choose a positive integer $N$ such that $1 / N<\varepsilon$. Pick any $x_{0} \in F_{n}$, then $x_{0} \in F_{n}$ for $n=1, \ldots, N$ so that $d\left(x_{0}, F_{n}\right)=0$ for $i=1, \ldots, N$ and $d \in \mathscr{D}$. Now for $n>N$ and $d \in \mathscr{D}$, $d\left(x_{0}, F_{n}\right) / n \leqslant 1 / n<1 / N<\varepsilon$. Thus $V\left(x_{0}\right)=\sup \left\{d\left(x_{0}, F_{n}\right) / n: d \in \mathscr{D}, n\right.$ $\geqslant 1\}<\varepsilon$. It follows that $\inf V(X)=0$. Next we claim that $P_{V}$ is positive definite $\bmod \Delta(X)$. Given $\varepsilon>0$ and $d \in \mathscr{D}$, we show that there exists $\delta(\varepsilon, d)>0$ such that $d(x, y) \geqslant \varepsilon$ implies $P(x, y) \geqslant \delta(\varepsilon, d)$. Indeed, given $\varepsilon>0$ and $d \in \mathscr{D}$, since $\left\{F_{n}\right\}$ contains small sets, there exists a positive integer $N$ such that the $d$-diameter $d\left(F_{N}\right)$ of $F_{N}$ is less than $\varepsilon / 3$. Choose $\delta(\varepsilon, d)$ $=\varepsilon / 3 N$. Then for any $x, y \in X$ with $d(x, y) \geqslant \varepsilon$, we must have either $d\left(x, F_{N}\right) \geqslant \varepsilon / 3$ or $d\left(y, F_{N}\right) \geqslant \varepsilon / 3$, so that $P(x, y) \geqslant \delta(\varepsilon, d)$. Hence $P$ is positive definite $\bmod \Delta(X)$. By hypothesis, $V(x)=0$ for some $x \in X$. Thus $d\left(x, F_{n}\right)=0$ for all $n \geqslant 1$ and $d \in \mathscr{Q}$. Since $\mathscr{D}$ is saturated, $x \in \operatorname{cl} F_{n}=F_{n}$ for all $n \geqslant 1$. Hence $\cap_{n=1}^{\infty} F_{n} \neq \varnothing$.

THEOREM 2. Let $(X$, Q) be a uniform space and $A$ a closed subset of $X$. Then $A$ is countably compact if and only if for every lower semicontinuous function $V$ of $X$ into $[0, \infty)$ with inf $V(A)=0, V(x)=0$ for some $x \in A$.

Proof. For the "if" part, let $\left\{A_{n}\right\}$ be a sequence of nonempty closed subsets of $A$ with the finite intersection property. We shall show that $\cap_{n=1}^{\infty} A_{n} \neq \varnothing$. Suppose to the contrary that $\cap_{n=1}^{\infty} A_{n}=\varnothing$. Without loss of generality, we may assume that $\left\{A_{n}\right\}$ is strictly decreasing. Define $G_{n}=X-A_{n}$ for $n=1,2$, $\ldots$ Then $\left\{G_{n}\right\}$ is a strictly increasing sequence of open sets in $X$ such that $\cup_{n=1}^{\infty} G_{n}=X$. For each $x \in X$, define 


$$
V(x)=\sup \left\{\chi_{G_{n}}(x) / n: n \geqslant 1\right\},
$$

where $\chi_{G_{n}}$ is the characteristic function of $G_{n}$ in $X$. Then $V$ is a lower semicontinuous function from $X$ into $[0, \infty)$. Since $\cup_{n=1}^{\infty} G_{n}=X, V(x)>0$ for all $x \in X$. On the other hand, for $n=1,2, \ldots$, there exists $x_{n}$ in $A_{n}$ such that $x_{n} \notin A_{n+1}$, and hence $V\left(x_{n}\right)=1 /(n+1)$. It follows that inf $V(A)=0$. By hypothesis, $V(x)=0$ for some $x \in A$. This is a contradiction. Therefore $\cap_{n=1}^{\infty} A_{n} \neq \varnothing$.

For the "only if" part, let $V$ be a lower semicontinuous function from $X$ into $[0, \infty)$ such that $\inf V(A)=0$. Since $A$ is countably compact and the restriction of $V$ to $A$ is also lower semicontinuous, there exists an $x$ in $A$ such that $V(x)=\min V(A)$, (see [1, Appendix] for a proof of this). Since inf $V(A)=0$, we must have $V(x)=0$.

Corollary. Let $(X$, थ) be a uniform space and let $A$ be a closed subset of $X$. Suppose that for every lower semicontinuous function $V$ of $X$ into $[0, \infty)$ such that $V$ is positive definite $\bmod A$ and $\inf V(A)=0, V(x)=0$ for some $x \in A$. Then $A$ is countably compact.

The above corollary generalizes Theorem 5 in [10], since it has weaker hypotheses and a stronger conclusion. By using Tietze's extension theorem, Dugundji obtained the above corollary for the case where $(X$, थ) is metrizable. It is now seen that the use of Tietze's extension theorem is not necessary. In view of the fact that the above corollary is a special case of the above theorem, the involving of positive definiteness in the above corollary does not seem natural. A probably more interesting problem is the converse of the above corollary. It is known that the converse of the above corollary is true if $A$ is compact [10]. This is due to the fact that for any open neighborhood $G$ of $A$, $U[A] \subset G$ for some $U \in \mathcal{Q}$.

\section{Applications.}

THEOREM 3. Let $(X$, थ) be a sequentially complete Hausdorff uniform space, $D$ a family of pseudometrics on $X$ which induces $Q$ and $T$ a continuous self-map on $X$. For each $d \in \mathscr{D}$, define

$$
V_{d}(x)=d(x, T(x)), \quad x \in X .
$$

Suppose that

(a) there exists a sequence $\left\{x_{n}\right\}$ in $X$ such that $\left\{\sup \left\{V_{d}\left(x_{n}\right): d \in \mathscr{D}\right\}\right\}$ converges to 0 ,

(b) for any $d \in \mathscr{D}$ and $\varepsilon>0$, there exists $\delta(\varepsilon, d)>0$ such that for any $x, y \in X, V_{d}(x)+V_{d}(y)<\delta(\varepsilon, d)$ implies $d(x, y)<\varepsilon$.

Then $T$ has a unique fixed point.

Proof. Define $V$ on $X$ by

$$
V(x)=\sup \left\{\min \left\{V_{d}(x), 1\right\}: d \in \mathscr{D}\right\}, \quad x \in X .
$$


Then $V$ is a lower semicontinuous function from $X$ into $[0, \infty)$ such that inf $V(x)=0$. For any $x, y \in X$, define $P_{V}(x, y)=V(x)+V(y)$. Then $P_{V}$ is positive definite $\bmod \Delta(X)$. By Theorem $1, V(x)=0$ for some $x \in X$. Thus $V_{d}(x)=0$ for all $d \in \mathcal{Q}$. Since $(X, \mathcal{Q})$ is Hausdorff, $x=T(x)$. Next let $x, y$ be fixed points of $T$. By (b), $d(x, y)<\varepsilon$ for all $d \in \mathscr{D}$ and $\varepsilon>0$. Since $(X, \mathscr{Q})$ is Hausdorff, $x=y$.

Now let $(X, \mathscr{Q})$ be a uniform space whose uniformity $\mathscr{Q}$ is induced by a family $\left\{d_{\lambda}\right\}_{\lambda \in \Gamma}$ of pseudometrics for $X$, and let $2^{X}$ be the family of all nonempty compact subsets of $X$. For each $\lambda \in \Gamma$, let $D_{\lambda}$ be the pseudometric for $2^{X}$ defined by

$$
D_{\lambda}(A, B)=\max \left\{\max \left\{d_{\lambda}(a, B): a \in A\right\}, \max \left\{d_{\lambda}(b, A): b \in B\right\}\right\}
$$

$$
A, B \in 2^{X} \text {. }
$$

Equip $2^{X}$ with the uniformity $\mathscr{V}$ induced by $\left\{D_{\lambda}\right\}_{\lambda \in \Gamma}$.

TheOREM 4. Let $(X, थ)$ be Hausdorff and sequentially complete and let $T: X \rightarrow 2^{X}$ be continuous. For each $\lambda \in \Gamma$, define

$$
V_{\lambda}(x)=d_{\lambda}(x, T(x)), \quad x \in X .
$$

Suppose that

(a) there exists a sequence $\left\{x_{n}\right\}$ in $X$ such that $\left\{\sup \left\{V_{\lambda}\left(x_{n}\right): \lambda \in \Gamma\right\}\right\}$ converges to 0 ,

(b) for each $\lambda \in \Gamma$ and $\varepsilon>0$, there exists $\delta(\varepsilon, \lambda)>0$ such that for any $x, y \in X, d_{\lambda}(x, T(x))+d_{\lambda}(y, T(y))<\delta(\varepsilon, \lambda)$ implies $d_{\lambda}(x, y)<\varepsilon$.

Then there exists an $x \in X$ such that $x \in T(x)$.

The above theorem generalizes Theorem 3 and its proof is left for the reader.

Sometimes a fixed point theorem corresponds to a coincidence theorem. Many of the coincidence results in [4] can be generalized to the present setting. We shall prove one and leave the rest (including statements and their proofs) to the interested reader.

THEOREM 5. Let $(X$, Q) be a sequentially complete Hausdorff uniform space whose uniformity $\mathcal{Q}$ is induced by a family $\mathscr{D}$ of pseudometrics for $X$ and let $(Y, \mathcal{V})$ be a Hausdorff uniform space. Let $S, T$ be continuous maps from $X$ into $Y$. Then $S \cap T \neq \varnothing(S$ and $T$ are considered as subsets of $X \times Y)$ if and only if there exists a lower semicontinuous function $V$ of $X \times Y$ into $[0, \infty)$ such that $V^{-1}(0) \subset S, \inf \{V(x, T(x)): x \in X\}=0$ and for any $d \in \mathscr{D}$ and $\varepsilon>0$, there exists $\delta(\varepsilon, d)>0$ such that for any $x, y \in X, V(x, T(x))+V(y, T(y))<$ $\delta(\varepsilon, d)$ implies $d(x, y)<\varepsilon$. Moreover, when such $V$ exists, and if $\left\{x_{n}\right\}$ is a sequence in $X$ such that $\left\{V\left(x_{n}, T\left(x_{n}\right)\right)\right\}$ converges to 0 , then $\left\{x_{n}\right\}$ converges to a point $x_{0} \in X$ with $\left(x_{0}, T\left(x_{0}\right)\right) \in T \cap S$.

Proof. For the "if" part, let $W$ be the function on $X$ defined by 


$$
W(x)=V(x, T(x)), \quad x \in X .
$$

Then $W$ is a lower semicontinuous function of $X$ into $[0, \infty)$ such that inf $W(X)=0$ and the function $P_{W}$ defined by

$$
P_{W}(x, y)=W(x)+W(y), \quad x, y \in X,
$$

is positive definite $\bmod \Delta(X)$. By Theorem $1, W\left(x_{0}\right)=0$ for some $x_{0} \in X$. Thus $\left(x_{0}, T\left(x_{0}\right)\right) \in V^{-1}(0)$ and hence $\left(x_{0}, T\left(x_{0}\right)\right) \in S \cap T$ as $V^{-1}(0) \subset S$. Now suppose $\left\{x_{n}\right\}$ is a sequence in $X$ such that $\left\{V\left(x_{n}, T\left(x_{n}\right)\right)\right\}$ converges to 0 . Then by the positive definiteness of $P_{W},\left\{d\left(x_{n}, x_{0}\right)\right\}$ converges to 0 for all $d \in \mathscr{D}$. Therefore $\left\{x_{n}\right\}$ converges to $x_{0}$.

For the "only if" part, let $\left(x_{0}, T\left(x_{0}\right)\right) \in S$. Let $\mathcal{E}$ be any family of pseudometrics for $Y$ which induces $\mathscr{V}$. For each $d_{1} \in \mathcal{D}$ and $d_{2} \in \mathcal{E}$, define $V_{d_{1}, d_{2}}: X \times Y \rightarrow[0, \infty)$ by

$$
V_{d_{1}, d_{2}}(x, y)=d_{1}\left(x, x_{0}\right)+d_{2}\left(y, S x_{0}\right), \quad(x, y) \in X \times Y
$$

Then $V_{d_{1}, d_{2}}$ is continuous on $X \times Y$ and $V_{d_{1}, d_{2}}\left(x_{0}, S x_{0}\right)=0$. Define $V: X \times Y$ $\rightarrow[0, \infty)$ by

$$
V(x, y)=\sup \left\{V_{d_{1}, d_{2}}(x, y) \wedge 1: d_{1} \in \mathscr{D}, d_{2} \in \mathcal{E}\right\}, \quad(x, y) \in X \times Y .
$$

Then $V$ is a lower semicontinuous function of $X \times Y$ into $[0,1]$ such that $V^{-1}(0)=\left\{\left(x_{0}, S x_{0}\right)\right\}$. Thus $V^{-1}(0) \subset S$ and $\inf \{V(x, T(x)): x \in X\}=0$. Finally, for any $d \in \mathscr{D}$ and $1>\varepsilon>0$, choose $\delta(\varepsilon, d)=\varepsilon$; then for any $x, y \in X$ with $d(x, y)>\varepsilon$, we have

$$
V(x, T(x))+V(y, T(y)) \geqslant d\left(x, x_{0}\right) \wedge 1+d\left(y, x_{0}\right) \wedge 1 \geqslant d(x, y) \wedge 1 \geqslant \varepsilon .
$$

\section{REFERENCES}

1. Jörg Blatter, Grothendieck spaces in approximations theory, Mem. Amer. Math. Soc. No. 120 (1972).

2. D. W. Boyd and J. S. W. Wong, On nonlinear contractions, Proc. Amer. Math. Soc. 20 (1969), 458-464. MR 39 \#916.

3. F. E. Browder, On the convergence of successive approximations for nonlinear functional equations, Nederl. Akad. Wetensch. Proc. Ser. A71 = Indag. Math. 30 (1968), 27-35. MR 37 \#5743.

4. J. Dugundji, Positive definite functions and coincidences, Séminaire de Mathématique Superieures (Fixed Point Theory and its Applications), Université de Montréal, June 1973.

5. M. Edelstein, On fixed and periodic points under contractive mapping, J. London Math. Soc. 37 (1962), 74-79. MR 24 \# A2936.

6. Chi Song Wong, Fixed point theorems for nonexpansive mappings, Ph.D. Thesis, Univ. of Illinois, Urbana, Illinois, 1969.

7. - Subadditive functions, Pacific J. Math. 36 (1971), 549-551. MR 43 \#4974. 
8. - Fixed point theorems for nonexpansive mappings, J. Math. Anal. Appl. 37 (1972), 142-150. MR 45 \#2691.

9. _ A fixed point theorem for a class of mappings, Math. Ann. 204 (1973), 97-103. MR 51 \#4206.

10. - On Dugundji's notion of positive definiteness, Proc. Amer. Math. Soc. 46 (1974), $443-450$.

Deparmant of Mathematics, Dalhousie University, Halifax, Nova Scotia, Canada

Departagnt of Mathematics, University of Windsor, Windsor, Ontario, Canada 